\title{
Similarity Evaluation of Different Origins and Species of Dendrobiums by GC-MS and FTIR Analysis of Polysaccharides
}

\author{
Nai-Dong Chen, ${ }^{1,2,3}$ Nai-Fu Chen, ${ }^{1}$ Jun Li, ${ }^{3}$ Cai-Yun Cao, ${ }^{1}$ \\ Jin-Mei Wang, ${ }^{1}$ and He-Ping Huang ${ }^{4}$ \\ ${ }^{1}$ College of Biotechnology and Pharmaceutical Engineering, West Anhui University, Lu'an 237012, China \\ ${ }^{2}$ West Anhui Biotechnology Research Center of Natural Medicine and Traditional Chinese Medicine, \\ West Anhui University, Lu'an 237012, China \\ ${ }^{3}$ School of Pharmacy, Anhui Medical University, Hefei 230032, China \\ ${ }^{4}$ School of Pharmacy, Anhui University of Chinese Medicine, Hefei 230012, China
}

Correspondence should be addressed to He-Ping Huang; hhp760222@126.com

Received 14 June 2015; Revised 16 August 2015; Accepted 27 August 2015

Academic Editor: Dimitrios Tsikas

Copyright (C) 2015 Nai-Dong Chen et al. This is an open access article distributed under the Creative Commons Attribution License, which permits unrestricted use, distribution, and reproduction in any medium, provided the original work is properly cited.

GC-MS method combined with FTIR techniques by the analysis of polysaccharide was applied to evaluate the similarity between wild (W) and tissue-cultured (TC) Dendrobium huoshanense (DHS), Dendrobium officinale (DO), and Dendrobium moniliforme (DM) as well as 3 wild Dendrobium spp.: Dendrobium henanense (DHN), Dendrobium loddigesii (DL), and Dendrobium crepidatum (DC). Eight monosaccharides involving xylose, arabinose, rhamnose, glucose, mannose, fructose, galactose, and galacturonic acid were identified in the polysaccharide from each Dendrobium sample while the contents of the monosugars varied remarkably across origins and species. Further similarity evaluation based on GC-MS data showed that the $r_{\text {cor }}$ values of different origins of DHS, DO, and DM were $0.831,0.865$, and 0.884 , respectively, while the $r_{\text {cor }}$ values ranged from 0.475 to 0.837 across species. FTIR files of the polysaccharides revealed that the similarity coefficients between $\mathrm{W}$ and TC-DHS, DO, and DM were $88.7 \%, 86.8 \%$, and $88.5 \%$, respectively, in contrast to the similarity coefficients varying from $57.4 \%$ to $82.6 \%$ across species. These results suggested that the structures of polysaccharides between different origins of the investigated Dendrobiums might be higher than what we had supposed.

\section{Introduction}

Dendrobium is the second largest genus in the family of Orchidaceae $[1,2]$. More than 70 species of Dendrobium are found in China [3]. The stems of some dendrobiums, including $D$. huoshanense, $D$. officinale, $D$. moniliforme, $D$. loddigesii, and $D$. crepidatum, are abundant in active compounds, especially in polysaccharides [4], and have been used as herbal medicines and nutraceutical products in China due to the potential benefiting effect to the stomach, moistening the lung, and immune-stimulating, antitumor, and antimutagenic activities [5-7]. Therefore, the demand for medicinal dendrobiums had constantly been increasing and resulted in overexploitation and depletion of some wild plant resources such as D. huoshanense, D. officinale, and D. moniliforme and left them critically endangered. In the traditional product region, tissue-cultured dendrobiums have already become the major resource of pharmaceutical dendrobiums. As plant amedica, it is extremely vital for tissue-cultured medicinal plants to obtain the ability to produce similar chemical components to their wild correspondences. Establishing a fast quality-identification method to evaluate the similarity of wild and tissue-cultured dendrobiums is a critical step for assurance of quality and safety in the TCM industry.

So far, the published phytochemical experiments have demonstrated that the bioactivities of polysaccharide were closely related to the level of monosaccharide compositions and the poly/monosaccharide ratio [8]. The analysis of monosaccharide compositions of polysaccharide is the most important step for further discovery of its physicochemical 
TABLE 1: The list of Dendrobium samples.

\begin{tabular}{lcccc}
\hline Samples & Abbreviation & Location & Gatherer & Voucher number \\
\hline Wild D. huoshanense & W-DHS & Huoshan, Anhui, China & Jun Dai & 201310HS0101Y \\
Tissue-cultured D. huoshanense & TC-DHS & Huoshan, Anhui, China & Jun Dai & 201310HS0101T \\
Wild D. officinale & W-DO & Huoshan, Anhui, China & Jun Dai & 201310HS0201Y \\
Tissue-cultured D. officinale & TC-DO & Huoshan, Anhui, China & Jun Dai & 201310HS0201T \\
Wild D. moniliforme & W-DM & Huoshan, Anhui, China & Jun Dai & 201310HS0301Y \\
Tissue-cultured D. moniliforme & TC-DM & Huoshan, Anhui, China & Jun Dai & 201310HS0301T \\
Wild D. henanense & W-DHN & Huoshan, Anhui, China & Jun Dai & 201310HS0401Y \\
Wild D. loddigesii & W-DL & Guilin, Guangxi, China & Jun Dai & 201310GX0501Y \\
Wild D. crepidatum & W-DC & Guilin, Guangxi, China & Jun Dai & 201310GX0501Y \\
\hline
\end{tabular}

properties, structure, and structure-bioactivity relationship and thus is an essential parameter for the quality evaluation of polysaccharide-based drugs and food ingredients with specific health enhancing functions $[3,9]$. Pharmacological studies revealed that, as one of the major bioactive components of Dendrobium plants, Dendrobium polysaccharide showed many beneficial effects on human physiology such as the immune-stimulatory properties $[10,11]$, immunoenhancing properties [9], and antitumor properties [12-14]. Therefore, it is essential to have information about whether the monosaccharide compositions and contents are similar among different origins and species of dendrobiums.

In this study, as one of our serial efforts on similarity evaluations of tissue-cultured dendrobiums and their wild correspondences, GC-MS and FTIR were applied to analyze the polysaccharides among different origins of D. huoshanense, D. officinale, and D. moniliforme as well as another 3 wild Dendrobium species. The fingerprints, based on GC-MS method combined with FTIR analysis, offered comprehensive information of the polysaccharides from Chinese herbals and would be useful for the quality assessment of medicinal dendrobiums of different origins and species and provide new insights into the utilization and conservation of rare and endangered medicinal plants by tissue-culture techniques.

\section{Experimental}

2.1. Plant Materials. The current season's vegetative stems of tissue-cultured and wild D. huoshanense, D. officinale, and D. moniliforme as well as wild $D$. henanense (Table 1) were collected in October 2013 from Huoshan County, Anhui Province, China. Wild D. loddigesii and D. crepidatum were collected in October 2013 from Guilin, Guangxi Province, China. All the plant materials were identified by Professor Nai-Fu Chen, Anhui Biotechnology Research Center of Plant Cell Engineering, Anhui Province, China. The voucher specimens (Table 1) are deposited at the Herbarium, College of Biotechnology and Pharmaceutical Engineering, West Anhui University, Anhui Province, China.

All the Dendrobium samples were dried in the sun and separately cut into small pieces, and then every $5.0 \mathrm{~g}$ of dried sample was investigated with the multistep analyzing procedure of polysaccharide by GC-MS and FTIR analysis.
2.2. Samples and Chemicals. The Dendrobium polysaccharides were prepared by hot-water extraction and ethanol precipitation. Briefly, the dried Dendrobium powders were defatted three times with $95 \%$ ethanol (w/v, $1: 50)$ at $80^{\circ} \mathrm{C}$, $3 \mathrm{~h}$ each, and then extracted three times with distilled water $(\mathrm{w} / \mathrm{v}, 1: 10)$ at $90^{\circ} \mathrm{C}, 3 \mathrm{~h}$ each time. The combined extracts were pooled and concentrated under a reduced pressure and then centrifuged at $4500 \mathrm{rpm}$ for $15.0 \mathrm{~min}$. The supernatant was collected and $100 \%$ ethanol was added slowly with stirring to precipitate the polysaccharide. The final ethanol concentration was adjusted to $76 \%$ and then kept at $4^{\circ} \mathrm{C}$ for $24 \mathrm{~h}$. The polysaccharide pellets were obtained by centrifugation at $5000 \mathrm{rpm}$ for $15 \mathrm{~min}$ and repeatedly washed sequentially with ethanol, acetone, and ether, respectively. The refined polysaccharide pellets were completely dissolved in appropriate volume of distilled water and repeatedly deproteinized by Sevag Method followed by filtration. Finally, the filtrate was lyophilized to yield diaphanous water-soluble Dendrobium polysaccharides.

Ethanol, acetone, ether, chloroform, n-butanol, methanol, hydrochloric acid, and pyridine were purchased from Sinopharm Chemical Reagent Co., Ltd. (Beijing, China). Hexamethyldisilazane and trimethylchlorosilane were bought from Aladdin Industrial Corporation (Shanghai, China). Hexane, myoinositol, xylose, arabinose, rhamnose, glucose, mannose, fructose, galactose, and galacturonic acid were bought from Sigma-Aldrich (Germany). Water was purified on a Milli-Q system (Millipore, Bedford, MA). The reagents from Sinopharm Chemical Reagent Co., Ltd., and Aladdin Industrial Corporation (Shanghai, China) are of analytical grade. The chemicals and reagents from Sigma-Aldrich are of chromatographic grade.

2.3. GC-MS Analysis. GC-MS analysis was performed with Trace 1300 Gas chromatograph coupled with ISQ mass spectrometer (Thermo Fisher Scientific, West Palm Beach, FL, USA) series equipment including TriPLUS RSH autosampler. The trimethylsilyl-ester O-methyl monosaccharides were separated on a TG-5 fused-silica capillary column $(30 \mathrm{~m} \times$ $0.25 \mathrm{~mm}, 0.25 \mu \mathrm{m}$ film thickness). The temperature program was $125^{\circ} \mathrm{C}$ to $155^{\circ} \mathrm{C}$ at $1.5^{\circ} \mathrm{C} / \mathrm{min}$, to $171^{\circ} \mathrm{C}$ at $0.8^{\circ} \mathrm{C} / \mathrm{min}$, and to $180^{\circ} \mathrm{C}$ at $9^{\circ} \mathrm{C} / \mathrm{min}$. The carrier gas was helium, at $1.0 \mathrm{~mL} / \mathrm{min}$, split ratio $20: 1$, and injector temperature $250^{\circ} \mathrm{C}$. The injection 
volume was $2.0 \mu \mathrm{L}$. The MS transfer line and ion source were at $250^{\circ} \mathrm{C}$ and $280^{\circ} \mathrm{C}$, respectively. The MS mode was electron ionization (EI). The mass range scanned was 50-350 amu.

2.4. Identification and Quantification of Monosaccharides by GC-MS. The GC-MS analysis was established according to the method proposed by Guadalupe et al. [15]. The monosaccharide composition was determined by GC-MS of the trimethylsilyl-ester O-methyl glycolsyl derivants (TMS) of monosaccharides obtained after acidic methanolyisis and derivatization. $20.0 \mathrm{mg}$ of polysaccharide of each Dendrobium sample was treated with $1.5 \mathrm{~mL}$ of the methanolysis reagent $(\mathrm{MeOH}$ containing $\mathrm{HCl} 0.5 \mathrm{M})$ in order to hydrolyse them to their corresponding methyl monosaccharides. The reaction was conducted in nitrogen atmosphere at $80^{\circ} \mathrm{C}$ for $16 \mathrm{~h}$ and thereafter the excess of reagent was removed using a stream of nitrogen gas. The conversion of the methyl glycosides to their trimethylsilyl (TMS) derivatives was performed by adding $0.5 \mathrm{~mL}$ of a mix of pyridine: hexamethyldisilazane : trimethylchlorosilane $(10: 2: 1 \mathrm{v} / \mathrm{v})$ to the dried material. The reaction was carried out at $80^{\circ} \mathrm{C}$ for $30 \mathrm{~min}$ and the reagent removed using a stream of nitrogen gas. A solution $(25 \mu \mathrm{L})$ of derivatized myoinositol was then added as internal standard and the derivative residues were extracted with $1 \mathrm{~mL}$ of hexane. GCMS was performed with $2.0 \mu \mathrm{L}$ of these solutions and samples were injected in duplicate. Different standard carbohydrates were also converted to their corresponding TMS derivatives and analyzed by GC-MS in order to obtain patterns for identification and the standard calibration curves.

2.5. FTIR Analysis. The FTIR analysis was performed as what we had reported in the previous paper $[16,17]$. FTIR spectra were recorded in the region of $4000-400 \mathrm{~cm}^{-1}$ on a Nicolet iS10 Fourier Transform Infrared Spectrum (Thermo Fisher Scientific, USA), equipped with a High-Performance Deuterated Triglycine Sulfate (DTGS) detector. The FTIR spectrometer was situated in an air-conditioned room $\left(25^{\circ} \mathrm{C}\right.$, $50 \%$ relative humidity).

The freeze-dried polysaccharides from the dendrobiums were separately powdered in a blender and screened using a 200-mesh sieve. Each sample was mixed uniformly with spectroscopic grade potassium bromide $(\mathrm{KBr})$ powder $(1: 100, \mathrm{w} / \mathrm{w})$ and then pressed into a pellet before infrared spectroscopic analysis. Each spectrum was calculated from a total of 36 coadded scans with a resolution of $4 \mathrm{~cm}^{-1}$, and pure $\mathrm{KBr}$ background spectrum was recorded before analysis of the samples. Each sample was scanned with five replicates. The scans of each sample were examined for consistence and the average spectrum of each sample was used for further analyses. Prior to data analysis, each of the averaged spectra was baseline corrected and smoothed with their absorbance normalized using the Spectrum for Windows software (Nicolet OMNIC, Thermo Fisher Scientific), so that the peak absorbance of the most intense band was set to unity.

2.6. Similarity Evaluation. The similarities of the polysaccharides based on the GC-MS data were evaluated by the correlation coefficient $\left(r_{\text {cor }}\right)$ calculated by included cosine

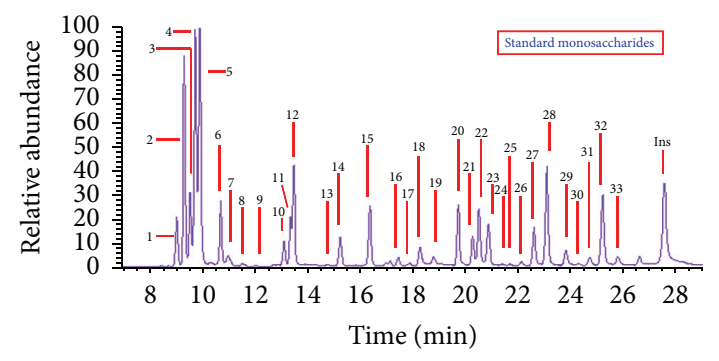

FIGURE 1: The TIC chromatograph of standard monosaccharide by TMS methyl method. Arabinose (peaks 1, 2, 5, 6, and 8), rhamnose (peaks 3, 4, 7, and 9), xylose (peaks 10-13), mannose (peaks 14-16 and 19), galactose (peaks 17, 18, 20, and 21), glucose (peaks 22, 28, 29 , and 32), galacturonic acid (peaks 23-26, 30, and 31), and fucose (peaks 27 and 33).

angle and using SPSS software Version 17.0 (CAMO Software AS, USA); the contents of the monosaccharide in the polysaccharides were selected as a measurement. The similarity evaluation based on the FTIR spectra was obtained using the algorithm of similarity coefficients which were automatically calculated by the software equipped in the Nicolet iS10 Fourier Transform Infrared Spectrum (Nicolet OMNIC, Thermo Fisher Scientific) $[16,17]$.

\section{Results and Discussion}

3.1. Identification and Quantification of Sugar Residues by GC-MS Detection. The monosaccharide compositions of the polysaccharides in the 9 Dendrobium samples were determined by GC-MS of their trimethylsilyl (TMS) derivatives and inositol was used as internal standard. The identification of the peaks was carried out by comparing retention times and mass spectra with those obtained by injections of pure standards. In our experiment, all the monosugars were converted to their corresponding TMS methyl glycoside derivatives and analyzed by GC-MS. Between four and seven peaks are obtained for each monosaccharide. The multiple peaks correspond to the TMS methyl glycoside derivatives of $\alpha$ - and $\beta$-anomers and the pyran-ring and furan-ring forms of the monosugars. Typical GC-MS chromatograms for the standard monosaccharides and the polysaccharides of the nine Dendrobium samples were shown in Figures 1 and 2. A total of eight kinds of monosugars including L-arabinose, rhamnose, D-xylose, D-galactose, D-glucose, D-mannose, $\mathrm{D}$-galacturonic acid, and fructose were identified in each of the nine investigated dendrobiums.

In order to quantify the monosaccharides in the GCMS chromatograms, calibration curves of the standard eight identified monosugars were detected by the ion monitoring (SIM) mode, selecting the appropriate number of ions for each compound $(\mathrm{m} / \mathrm{z})$ in one segment from 7 to $32 \mathrm{~min}$ : $\mathrm{D}$-galacturonic acid, L-rhamnose, fucose, D-galactose, Dglucose, D-mannose, and D-xylose with 204 ions and Larabinose with 217 ions. For all the spectra, these ions were their base peaks and obtained the most ion abundance and were selected for recording SIM mode chromatograms. 

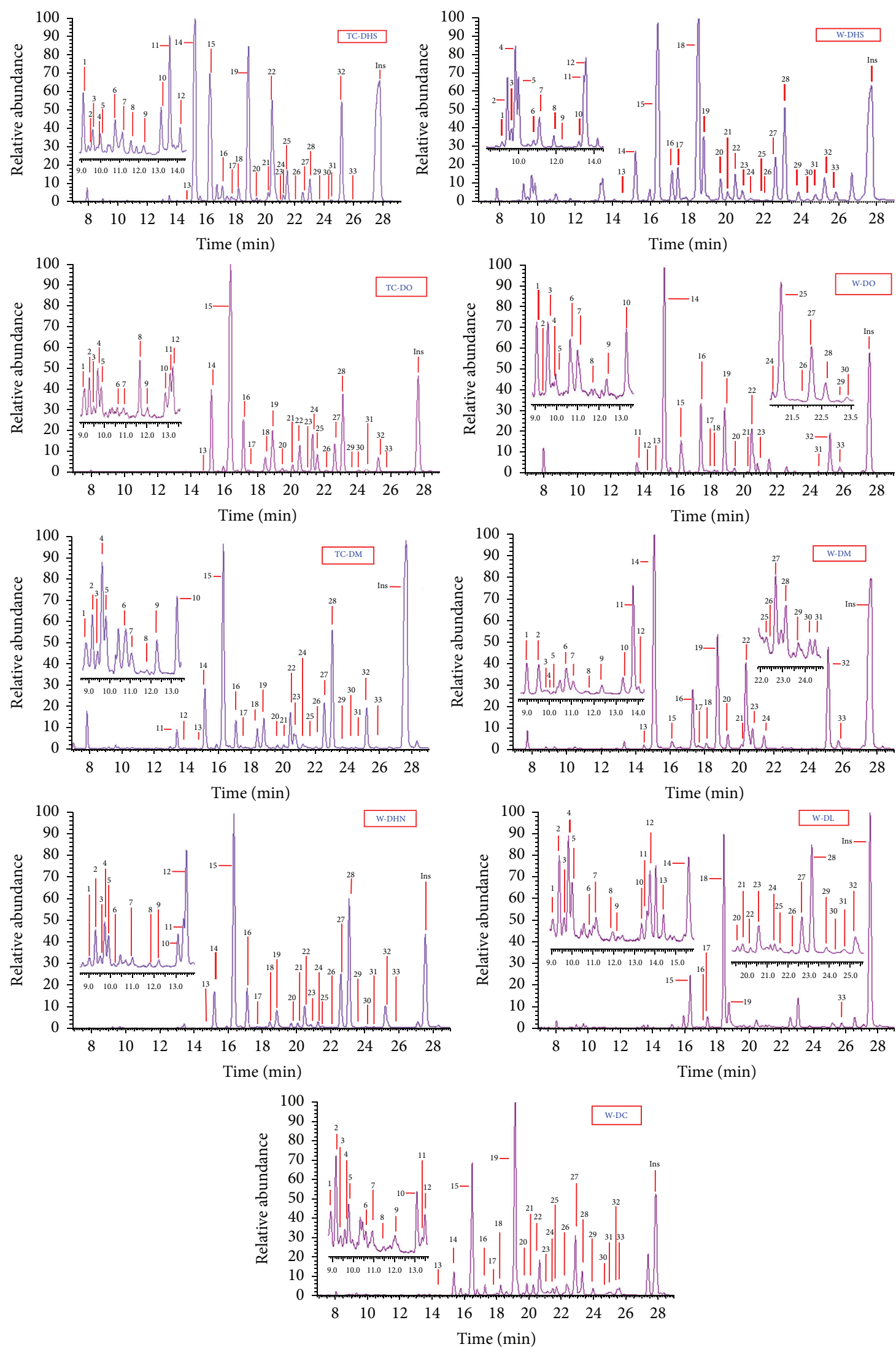

FIGURE 2: GC-MS comparison of the monosaccharides in the polysaccharides from the nine Dendrobium samples by TMS methyl method. Arabinose (peaks 1, 2, 5, 6, and 8), rhamnose (peaks 3, 4, 7, and 9), xylose (peaks 10-13), mannose (peaks 14-16 and 19), galactose (peaks 17, 18, 20, and 21), glucose (peaks 22, 28, 29, and 32), galacturonic acid (peaks 23-26, 30, and 31), and fucose (peaks 27 and 33 ). 
TABLE 2: Calibration curve data, calculated detection (LOD), and quantification (LOQ) limits and precision (repeatability and reproducibility) of the monosaccharide standards detected by GC-MS.

\begin{tabular}{|c|c|c|c|c|c|c|c|c|}
\hline Compounds & Equation & $\mathrm{Sd}_{\text {slope }}$ & $\begin{array}{c}\text { Correlation } \\
\text { coefficient }\left(r^{2}\right)\end{array}$ & $\begin{array}{c}\text { Linear } \\
\text { range }(\mu \mathrm{g})\end{array}$ & LOD $(\mu \mathrm{g})$ & LOQ $(\mu \mathrm{g})$ & Reproducibility (\%) & Repeatability (\%) \\
\hline Arabinose & $A=0.607 C$ & 0.008 & 0.985 & $0.5-1600$ & 0.2 & 0.8 & 12 & 6 \\
\hline Rhamnose & $A=0.652 C$ & 0.090 & 0.975 & $0.5-1600$ & 0.2 & 0.5 & 9 & 11 \\
\hline Xylose & $A=0.547 C$ & 0.007 & 0.989 & $0.5-600$ & 0.1 & 0.5 & 7 & 6 \\
\hline Mannose & $A=0.811 C$ & 0.092 & 0.993 & $2.5-1500$ & 0.1 & 0.6 & 7 & 3 \\
\hline Galactose & $A=0.392 C$ & 0.010 & 0.987 & $1.5-1500$ & 0.3 & 0.7 & 7 & 5 \\
\hline Glucose & $A=0.913 C$ & 0.023 & 0.998 & $1.0-1600$ & 0.1 & 1.8 & 7 & 5 \\
\hline $\begin{array}{l}\text { Galacturonic } \\
\text { acid }\end{array}$ & $A=0.238 C$ & 0.005 & 0.977 & $4.0-1300$ & 1.0 & 5.0 & 9 & 7 \\
\hline Fucose & $A=0.566 C$ & 0.010 & 0.969 & $0.5-550$ & 0.4 & 1.2 & 12 & 9 \\
\hline Inositol & $A=0.954 C$ & 0.035 & 0.993 & $1.0-1500$ & 0.2 & 1.5 & 10 & 1 \\
\hline
\end{tabular}

TABLE 3: Monosaccharide compositions and contents (\%) of the polysaccharides from the nine Dendrobium samples detected by GC-MS analysis of their TMS derivatives.

\begin{tabular}{lccccccccc}
\hline Com. & TC-DHS & W-DHS & TC-DO & W-DO & TC-DM & W-DM & W-DHN & W-DL & W-DC \\
\hline Arabinose & $0.6 \pm 0.1$ & $2.8 \pm 0.4$ & $0.1 \pm 0.0$ & $0.2 \pm 0.1$ & $0.7 \pm 0.1$ & $0.6 \pm 0.1$ & $0.4 \pm 0.1$ & $1.2 \pm 0.1$ & $0.5 \pm 0.1$ \\
Rhamnose & $0.3 \pm 0.1$ & $2.7 \pm 0.3$ & $0.1 \pm 0.0$ & $0.2 \pm 0.0$ & $0.7 \pm 0.1$ & $0.5 \pm 0.1$ & $0.4 \pm 0.1$ & $1.1 \pm 0.1$ & $0.6 \pm 0.1$ \\
Xylose & $0.3 \pm 0.1$ & $3.0 \pm 0.2$ & $0.1 \pm 0.1$ & $0.2 \pm 0.1$ & $0.3 \pm 0.1$ & $0.2 \pm 0.1$ & $1.0 \pm 0.1$ & $0.9 \pm 0.2$ & $0.5 \pm 0.1$ \\
Mannose & $62.0 \pm 1.2$ & $32.2 \pm 1.1$ & $65.6 \pm 2.3$ & $59.5 \pm 1.5$ & $48.5 \pm 1.3$ & $60.0 \pm 1.7$ & $50.6 \pm 1.4$ & $17.9 \pm 0.9$ & $59.7 \pm 2.1$ \\
Galactose & $2.2 \pm 0.9$ & $25.8 \pm 1.3$ & $3.3 \pm 0.3$ & $13.8 \pm 0.7$ & $3.9 \pm 0.1$ & $2.3 \pm 0.2$ & $2.5 \pm 0.2$ & $41.8 \pm 1.7$ & $3.8 \pm 0.2$ \\
Glucose & $27.0 \pm 1.1$ & $14.6 \pm 0.9$ & $17.9 \pm 1.1$ & $18.4 \pm 1.2$ & $29.8 \pm 0.9$ & $30.9 \pm 1.3$ & $29.9 \pm 1.3$ & $11.3 \pm 0.3$ & $10.4 \pm 0.6$ \\
Galacturonic acid & $4.1 \pm 0.8$ & $3.3 \pm 0.2$ & $9.6 \pm 0.5$ & $0.1 \pm 0.0$ & $5.1 \pm 0.5$ & $2.3 \pm 0.1$ & $2.6 \pm 0.2$ & $4.8 \pm 0.3$ & $6.5 \pm 0.3$ \\
Fucose & $1.2 \pm 0.1$ & $11.9 \pm 0.7$ & $4.5 \pm 0.4$ & $4.1 \pm 0.4$ & $10.2 \pm 0.8$ & $1.8 \pm 0.1$ & $10.1 \pm 0.5$ & $16.7 \pm 1.1$ & $16.7 \pm 0.8$ \\
\hline
\end{tabular}

Values are means \pm standard deviations $(n=3)$.

The features of the GC-MS detection are listed in Table 2, which includes the equation, the slope with its standard deviation, the correlation coefficients $\left(r^{2}\right)$, the linear range, and the limit of detection (LOD) and the limit of quantification (LOQ) for the carbohydrate standards. The detection limits of each monosaccharide were obtained by injecting a standard mixture of derivatives as mentioned above in the derivation procedure, followed by the comparison of peak height with a signal-to-noise ratio $(\mathrm{S} / \mathrm{N})$ of three assigned limits of detection (LOD) and a signal to noise of ten assigned limits of quantification (LOQ). The features of the GCMS method were established after a linearity study using solutions of standard carbohydrates. The analyte-to-internalstandard-peak-area ratio was used as analytical signal for constructing the calibration graphs. The limit of detection was calculated as the concentration of a signal to noise of three and the limit of quantification from the signal to noise of ten.

The correlation coefficients obtained from the calibration curves were all higher than $0.96(p<0.001)$. These curves were, therefore, considered to be linear for the range of concentrations studied (to $1500-1600 \mu \mathrm{g}$ for arabinose, rhamnose, mannose galactose, and glucose, 550-600 $\mu \mathrm{g}$ for fucose and xylose, and $1300 \mu \mathrm{g}$ for galacturonic acid). The limits of detection and quantification were good and in all the cases they were below the values obtained for the monosaccharides present in the nine Dendrobium samples. The validation of the proposed method was carried out by analyzing Dendrobium samples. The precision of the GC-MS method was checked in terms of repeatability and reproducibility (Table 2) by means of an analysis of variance (ANOVA). Repeatability was evaluated by the analysis of six replicates of the same Dendrobium under normal operating conditions and it was expressed as relative standard deviation values. The reproducibility of the method was evaluated by analysis of three batches of the investigated dendrobiums and was expressed as the relative standard deviation values. As described in the Experimental, samples were defatted, extracted by hot water, precipitated by ethanol, and deproteinized by Sevag Method. The collected residues were freeze-dried, methylated, derivatizated, and submitted to GC-MS analysis to calculate the amount of carbohydrates. Both repeatability and reproducibility values were good taking into account the fact that a multistep procedure was performed with values ranging between 1 and $11 \%$ and 7 and $12 \%$, respectively. The results of the analysis on the monosaccharide contents in the polysaccharides from the nine investigated dendrobiums were shown in Table 3, respectively.

All the investigated dendrobiums were detected to obtain the same monosaccharide compositions in our experimental 
TABLE 4: The correlation coefficients of the nine Dendrobium samples.

\begin{tabular}{|c|c|c|c|c|c|c|c|c|c|}
\hline & TC-DHS & W-DHS & TC-DO & W-DO & TC-DM & W-DM & W-DHN & W-DL & W-DC \\
\hline TC-DHS & 1 & 0.831 & 0.780 & 0.764 & 0.775 & 0.795 & 0.777 & 0.475 & 0.719 \\
\hline W-DHS & & 1 & 0.738 & 0.764 & 0.736 & 0.726 & 0.732 & 0.787 & 0.750 \\
\hline TC-DO & & & 1 & 0.865 & 0.837 & 0.760 & 0.738 & 0.494 & 0.765 \\
\hline W-DO & & & & 1 & 0.729 & 0.754 & 0.731 & 0.683 & 0.735 \\
\hline TC-DM & & & & & 1 & 0.884 & 0.797 & 0.521 & 0.795 \\
\hline W-DM & & & & & & 1 & 0.784 & 0.479 & 0.794 \\
\hline W-DHN & & & & & & & 1 & 0.502 & 0.701 \\
\hline W-DL & & & & & & & & 1 & 0.243 \\
\hline W-DC & & & & & & & & & 1 \\
\hline
\end{tabular}

conditions while the sugar contents differed remarkably among different origins and species. For example, the contents of mannose and glucose in the polysaccharide from TCDHS were $62.0 \pm 1.2$ and $27.0 \pm 1.1 \%$, nearly twice those from W-DHS $(32.2 \pm 1.1$ and $14.6 \pm 0.9 \%$, resp.) and about three times as much as those from W-DL $(17.9 \pm 0.9 \%$ and $11.3 \pm 0.3 \%$, resp.) (Table 3 ). Total $9.6 \pm 0.5 \%$ of galacturonic acid was detected in the polysaccharide from TC-DO while only $0.1 \pm 0.0 \%$ of galacturonic acid was checked out in W-DO, and the content of galacturonic acid in the polysaccharides from TC-DM was more than twice as much as that in its wild correspondence. The contents of galactose in W-DHS and $\mathrm{W}$-DO were $25.8 \pm 1.3 \%$ and $13.8 \pm 0.7 \%$, respectively, obviously higher than those in TC-DHS $(2.2 \pm 0.9 \%)$ and TCDO $(3.3 \pm 0.3 \%)$. Thus, the nine Dendrobium samples might be discriminated approximately.

It was noted that a high content of mannose was detected in the polysaccharides from the investigated dendrobiums (with the mannose ratios $17.0-67.9 \%$, Table 3). The contents of glucose, galactose, and fucose were also very high and the total content of the three monosugars in the polysaccharides was $27.5 \%$ (TC-DO, Table 3) to $71.8 \%$ (W-DL, Table 3 ). These results indicated that the Dendrobium polysaccharides were heteropolysaccharide including mannose, glucose, galactose, and fucose as major components and arabinose, xylose, rhamnose, and galacturonic acid as minor components.

The above analysis indicated that the structures of the polysaccharides between the investigated tissue-cultured dendrobiums and their wild correspondences might be different. Furthermore, the variations of the contents of monosaccharides might provide new approaches in the discrimination and identification of the nine dendrobiums.

3.2. Similarity Analysis of GC-MS Fingerprint. To evaluate the similarity of chemical compositions of different origins and species of dendrobiums directly, the correlation coefficients $\left(r_{\text {cor }}\right)$ were calculated by cosine angle method based on the data of monosugars in polysaccharides and were used as similarity measure. As it was shown in Table $4, r_{\text {cor }}$ values between $\mathrm{W}$ - and TC-DHS, W- and TC-DO, and Wand TC-DM were only $0.831,0.865$, and 0.884 , respectively, and $r_{\text {cor }}$ values ranged from 0.475 to 0.837 across species. In the term of the compositions and the contents of the monosaccharides, the similarities between different origins of

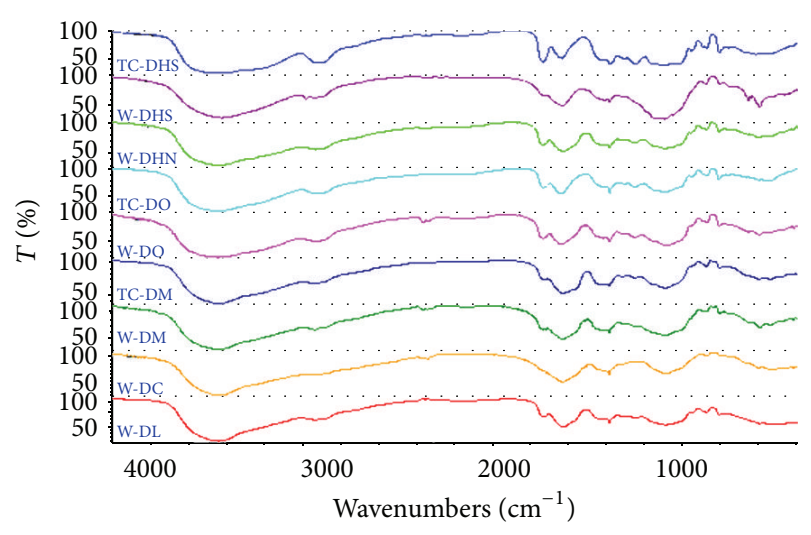

FIGURE 3: The FTIR spectra of the polysaccharides of seven Dendrobium samples in the ranges of $4000-400 \mathrm{~cm}^{-1}$.

Dendrobium samples were higher than those across species, and the differences of the polysaccharide in tissue-cultured dendrobiums and their wild correspondences might be more than what we had proposed though they were cultivated in the same conditions.

3.3. FTIR Spectroscopy Analysis of Polysaccharides. Fourier transform infrared spectroscopy (FTIR) is an important method to predict the structures of natural macromolecules such as polysaccharide and is an alternative method for TCM discrimination respecting the notion that the macrofingerprint features of FTIR spectra are consistent with the holistic theory of TCM. Considering the notion that the similarity analysis based on the monosugar contents might not effectively reflect the overall structural variations among the polysaccharides from different origins and species, the FTIR spectrum was investigated to further evaluate the similarity among the nine Dendrobium samples. Figure 3 showed the IR spectra of the nine Dendrobium polysaccharides, respectively. All samples' IR spectrum had a broad stretching intense characteristic peak at around $3425 \pm 4 \mathrm{~cm}^{-1}$ for $\mathrm{OH}$ and a weak peak at around $2935 \pm 4 \mathrm{~cm}^{-1}$ for $\mathrm{CH}$ bond. The peaks at around $1650 \pm 4 \mathrm{~cm}^{-1}$ and $1380 \pm 4 \mathrm{~cm}^{-1}$ were attributed to the bending vibration of $\mathrm{C}=\mathrm{O}$. The characteristic peak around $860 \pm 4 \mathrm{~cm}^{-1}$ implied the presence of $\beta$-D-glycosidic linkages in the polysaccharides. 
TABLE 5: The similarity coefficients of the FTIR of the polysaccharides from the nine dendrobiumsin the ranges of $4000-400 \mathrm{~cm}^{-1}(\%)$.

\begin{tabular}{|c|c|c|c|c|c|c|c|c|c|}
\hline Similarity coefficients & TC-DHS & W-DHS & TC-DO & W-DO & TC-DM & W-DM & W-DHN & W-DL & W-DC \\
\hline TC-DHS & 100 & 88.7 & 77.9 & 75.7 & 75.0 & 79.4 & 77.5 & 61.1 & 70.1 \\
\hline W-DHS & & 100 & 62.2 & 67.9 & 75.6 & 82.3 & 74.9 & 68.1 & 67.1 \\
\hline TC-DO & & & 100 & 86.8 & 83.7 & 77.9 & 80.1 & 58.6 & 81.9 \\
\hline $\mathrm{W}-\mathrm{DO}$ & & & & 100 & 80.1 & 77.8 & 76.1 & 71.2 & 78.4 \\
\hline TC-DM & & & & & 100 & 88.5 & 82.6 & 63.4 & 73.1 \\
\hline W-DM & & & & & & 100 & 80.5 & 68.8 & 76.2 \\
\hline W-DHN & & & & & & & 100 & 57.4 & 78.5 \\
\hline W-DL & & & & & & & & 100 & 77.4 \\
\hline W-DC & & & & & & & & & 100 \\
\hline
\end{tabular}

3.4. Similarity Analysis of FTIR Fingerprint. The similarity analysis based on the IR spectra of the polysaccharides from the 9 Dendrobium samples was shown in Table 5. The similarities between different origins of DHS, DO, and DM were only $86.8-88.7 \%$ though they were higher than those between species of the investigated dendrobiums, consistent with the results of GC-MS analysis, further confirming that the differences of the polysaccharides structure might exist between tissue-cultured dendrobiums and their wild correspondences.

\section{Conclusion}

In conclusion, the results obtained in this study can provide comprehensive evaluation for quality between tissuecultured and wild dendrobiums and offer an optimized evaluation method for medicinal herb quality control. A total of 8 monosugars with different contents were identified in the polysaccharide from each of the nine Dendrobium samples. The similarity evaluation based on GC-MS data showed that $r_{\text {cor }}$ values of different origins of $D$. huoshanense, D. officinale, and D. moniliforme were $0.831,0.865$, and 0.884 , respectively, while $r_{\text {cor }}$ values ranged from 0.475 to 0.837 across species. The similarity evaluation based on FTIR files of the polysaccharides showed that the similarity coefficients between W and TC D. huoshanense, D. officinale, and D. moniliforme were $88.7 \%, 86.8 \%$, and $88.5 \%$, respectively, in contrast to the similarity coefficients varying from $57.4 \%$ to $82.6 \%$ across species. The results revealed that although the genetic information was the same between tissue-cultured dendrobiums and their wild correspondences and was very similar among the different Dendrobium spp., the monosaccharide compositions as well as the whole structure of the polysaccharides might be quite different between origins and across species. How to keep the similarity of the main bioactive components including polysaccharide between the tissue-cultured medicinal plants and their wild correspondences would be a great challenge and bottleneck in the conversation and utilization of the in-danger Dendrobium species such as $D$. huoshanense using tissue-culture technology.

\section{Conflict of Interests}

The authors declare that there is no conflict of interests regarding the publication of this paper.

\section{Acknowledgments}

This work was supported by the National Natural Science Foundation of China (NSFC no. 81274021, NSFC no. 81573536), the Postdoctoral Science Fund of China (2014M551791), China, the Provincial Level Nature Science Foundation of Anhui Education Department (KJ2015ZD43), the Postdoctoral Science Foundation of Anhui Province, and the Research-Based Learning Program of West Anhui University (20141037610, AH201410376003, and AH201410376005).

\section{References}

[1] W. Xu, F. Zhang, B.-B. Lu et al., "Development of novel chloroplast microsatellite markers for Dendrobium officinale, and cross- amplification in other Dendrobium species (Orchidaceae)," Scientia Horticulturae, vol. 128, no. 4, pp. 485-489, 2011.

[2] D. Puchooa, "Comparison of different culture media for the in vitro culture of Dendrobium (Orchidaceae)," International Journal of Agriculture and Biology, vol. 6, pp. 884-888, 2004.

[3] X. K. Wang and T. F. Zhao, "A discussion of the chemical constituents of Dendrobium plants and of the Chinese herbal drug Shi-hu," International Journal of Oriental Medicine, vol. 15, pp. 146-155, 1990.

[4] X. M. Chen and S. X. Guo, "Advances in the research of constituents and pharmacology of Dendrobium," Natural Product Research and Development, vol. 13, no. 1, pp. 70-75, 2000.

[5] J.-H. Wang, J.-P. Luo, X.-Q. Zha, and B.-J. Feng, "Comparison of antitumor activities of different polysaccharide fractions from the stems of Dendrobium nobile Lindl," Carbohydrate Polymers, vol. 79, no. 1, pp. 114-118, 2010.

[6] T. B. Ng, J. Liu, J. H. Wong et al., "Review of research on Dendrobium, a prized folk medicine," Applied Microbiology and Biotechnology, vol. 93, no. 5, pp. 1795-1803, 2012.

[7] J. Liu, X. Chen, W. Yang, W. Liu, and T. Jiang, "Study on establishment of RP-HPLC and GC-MS fingerprints for wild germplasm resource of Ophiopogon japonicus in Sichuan and hierarchical clustering analysis," Zhongguo Zhongyao Zazhi, vol. 35, no. 20, pp. 2726-2730, 2010.

[8] M. Zhang, S. W. Cui, P. C. K. Cheung, and Q. Wang, "Antitumor polysaccharides from mushrooms: a review on their isolation process, structural characteristics and antitumor activity," Trends in Food Science and Technology, vol. 18, no. 1, pp. 4-19, 2007. 
[9] T. Y. Cai, Q. L. Liu, D. Li, A. Z. Chen, B. H. Huang, and S. J. Cheng, "Effects of Dendrobium officinale polysaccharides on the activities of T lymphocytes and macrophages," Academic Journal of Sun Yat-Sen University of Medical Sciences, vol. 10, pp. 66-67, 1989.

[10] D. Grindlay and T. Reynolds, "The Aloe vera phenomenon: a review of the properties and modern uses of the leaf parenchyma gel," Journal of Ethnopharmacology, vol. 16, no. 2-3, pp. 117-151, 1986.

[11] T. Reynolds and A. C. Dweck, "Aloe vera leaf gel: a review update," Journal of Ethnopharmacology, vol. 68, no. 1-3, pp. 3-37, 1999.

[12] T. G. He, L. T. Yang, Y. R. Li, C. Q. Wang, and J. S. Hu, "Effects of the polysaccharides DCPPla-1 from suspension-cultured protocorms of Dendrobium candidumon oxygen radical and lipid peroxidation," Natural Products Research and Development, vol. 19, pp. 410-414, 2007.

[13] A. X. Luo and Y. J. Fan, "Immune stimulating activity of water-soluble polysaccharide fractions from Dendrobium nobile Lindl," African Journal of Pharmacy and Pharmacology, vol. 5, no. 5, pp. 625-631, 2011.

[14] H. Y. Zhang, G. H. Dai, C. Ma, and F. Yang, "Effects of Dendrobium officinale polysaccharides on the immune functions of Sarcoma 180-bearing mice," Zhejiang Journal of Traditional Chinese Medicine, vol. 44, pp. 380-381, 2009.

[15] Z. Guadalupe, O. Martínez-Pinilla, Á. Garrido, J. D. Carrillo, and B. Ayestarán, "Quantitative determination of wine polysaccharides by gas chromatography-mass spectrometry (GC-MS) and size exclusion chromatography (SEC)," Food Chemistry, vol. 131, no. 1, pp. 367-374, 2012.

[16] N.-D. Chen, H. Chen, J. Li, M.-M. Sang, S. Ding, and H. Yu, "Discrimination and similarity evaluation of tissue-cultured and wild Dendrobium species using Fourier transform infrared spectroscopy," Journal of Molecular Structure, vol. 1086, pp. 255265, 2015.

[17] N. D. Chen, N. F. Chen, J. Li, C. Cao, and J. Wang, "Rapid authentication of different ages of tissue-cultured and wild Dendrobium huoshanense as well as wild Dendrobium henanense using FTIR and 2D-COS IR," Journal of Molecular Structure, vol. 1101, pp. 101-108, 2015. 

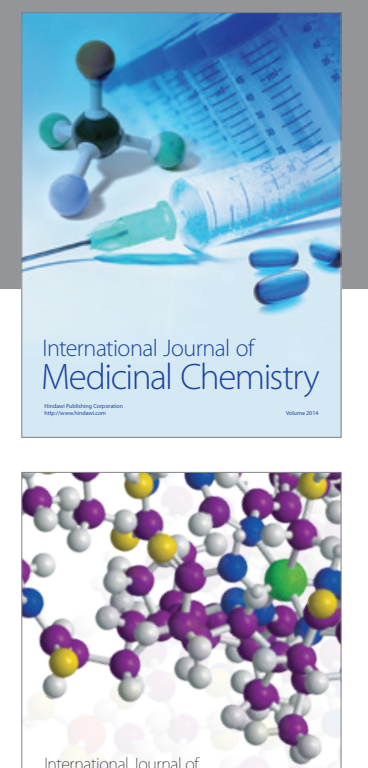

\section{Carbohydrate} Chemistry

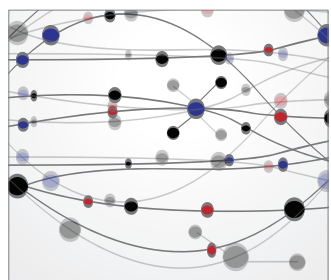

The Scientific World Journal
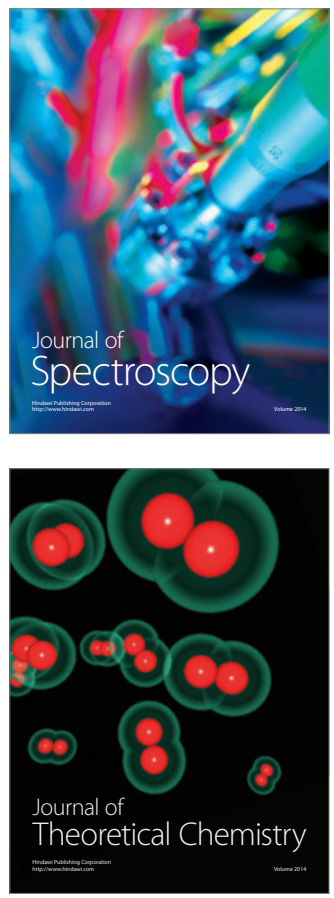
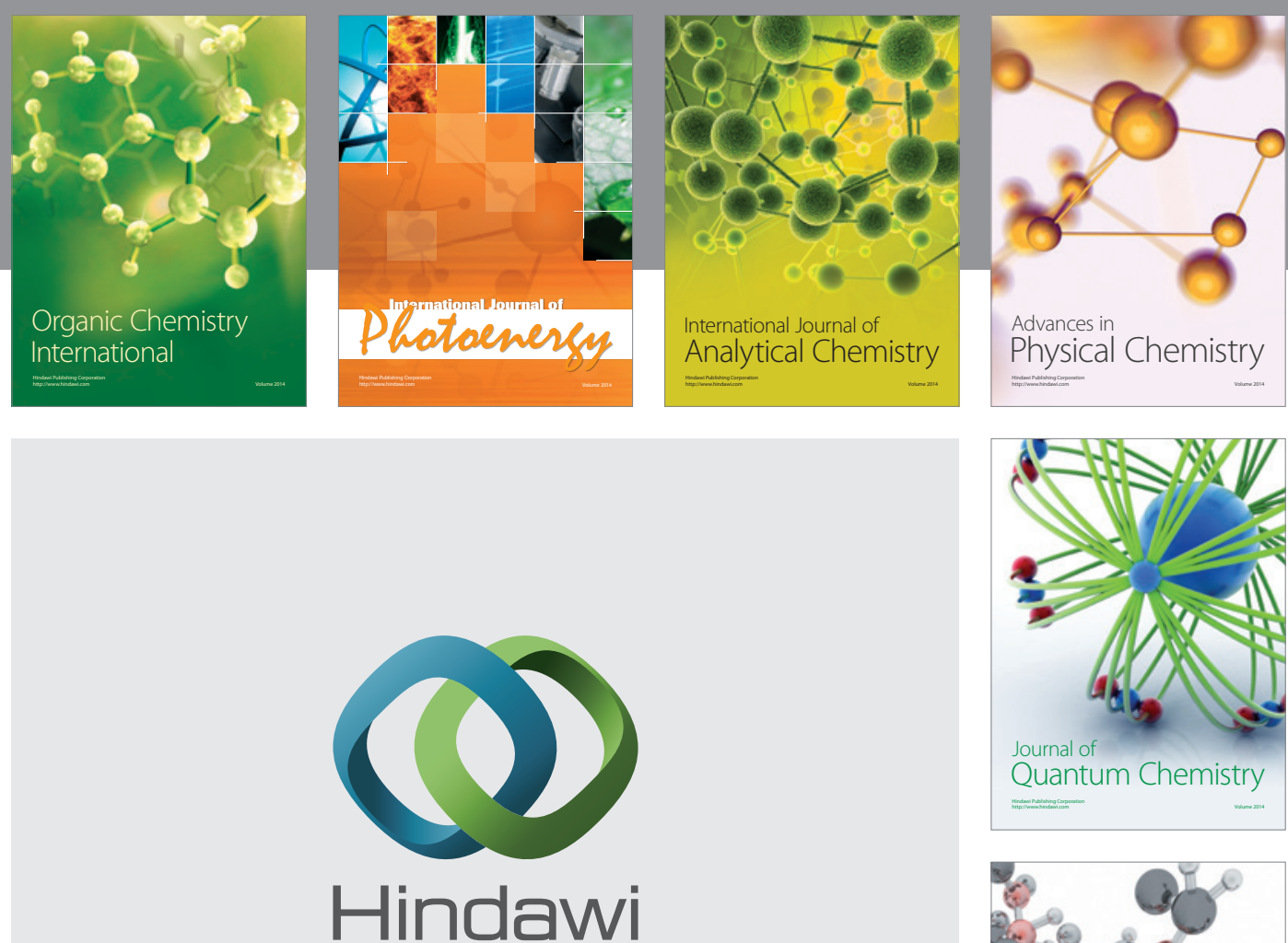

Submit your manuscripts at

http://www.hindawi.com

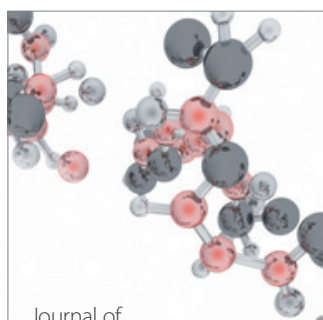

Analytical Methods

in Chemistry

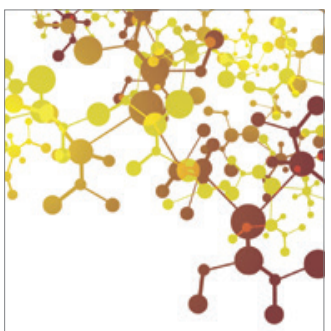

Journal of

Applied Chemistry

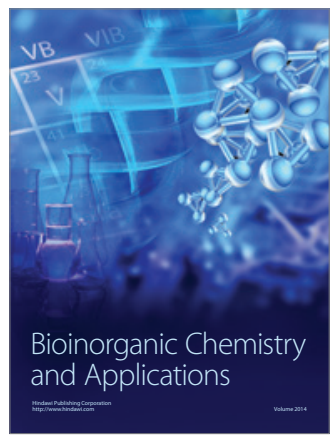

Inorganic Chemistry
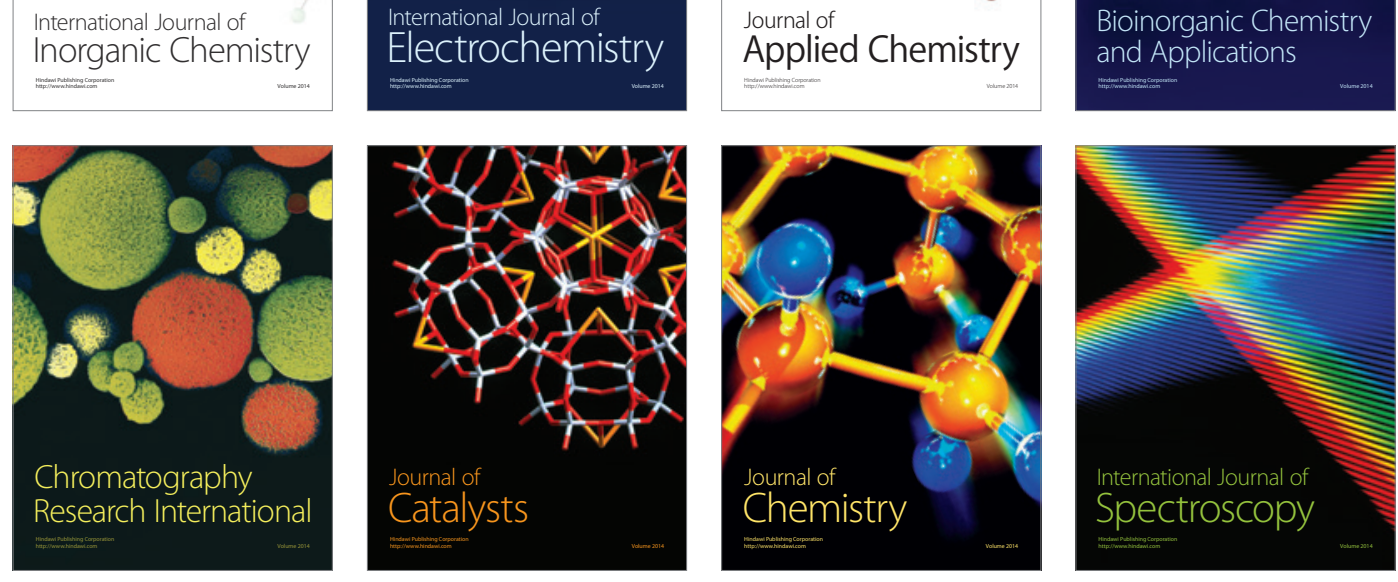